\title{
Ethical aspects of cloning techniques
}

\author{
Advisers to the President of the European Commission on the Ethical Implications of Biotechnology
}

\section{Editor's note}

Following a request from the President of the European Commission, M Facques Santer, the Group of Advisers on the Ethical Implications of Biotechnology adopted an Opinion on 28 May 1997, examining the ethical consequences of cloning. The Opinion is published in its entirety here.

The Group of Advisers on the Ethical Implications of Biotechnology (GAEIB) to the European Commission,

Having regard to the commission's request of 28 February 1997 for an Opinion on the ethical implications of cloning techniques, namely animal cloning and application potential to human beings,

Having regard to the Treaty on European Union, namely the article F 2 of the Common Provisions and the annexed Declaration no 24 on the Protection of Animals,

Having regard to the council directive 86/609/EEC regarding the protection of animals used for experimental and other scientific purposes,

Having regard to the council directive 90/220/EEC, regarding the deliberate release into the environment of genetically modified organisms,

Having regard to the council and European parliament decision no 1110/94/EC of 26 April 1994 adopting the 4th Framework Programme,

Having regard to the resolutions of the European parliament, namely the resolutions of 16 March 1989 on the ethical and legal problems of genetic engineering, of 28 October 1993 on the cloning of the human embryo and of 12 March 1997 on cloning,

Having regard to the European Conventions of the Council of Europe for the protection of animals kept for farming purposes (1976-EST 87) and in particular the protocol of amendment thereto, and for the protection of vertebrate animals used for experimental or other scientific purposes (1986-EST 123), Having regard to the Convention of the Council of Europe on Human Rights and Biomedicine, signed on 4th April 1997,

Having regard to the United Nations Convention on
Biodiversity of 6 June 1992, ratified by the European Union on 25 October 1993, Having regard to the draft declaration of UNESCO, Universal Declaration on the Human Genome and Human Rights, of 20 December 1996,

Having regard to the hearings, organised on 18 April 1997 by the GAEIB with members of the European parliament and commission, international organisations (WHO and UNESCO), researchers, industry, representatives of consumers, patients and environment organisations and animal protection associations.

The following points aim to shed light on the cloning debate by giving information on the scientific aspects of cloning and the ethical problems relating to them.

\section{Whereas}

1.1 Cloning is the process of producing "genetically identical" organisms. It may involve division of a single embryo, in which case both the nuclear genes and the small number of mitochondrial genes would be "identical", or it may involve nuclear transfer, in which case only the nuclear genes would be "identical". But genes may be mutated or lost during the development of the individual: the gene set may be identical but it is unlikely that the genes themselves would ever be totally identical. In the present context, we use the term "genetically identical" to mean "sharing the same nuclear gene set".

1.2 It is inherent in the process of sexual reproduction that the progeny differ genetically from one another. In contrast, asexual reproduction (cloning) produces genetically identical progeny. This is a common form of reproduction in plants, both in nature and in the hands of plant breeders and horticulturists. Once a desired combination of characteristics has been achieved asexual reproduction is the best way of preserving it. Asexual reproduction is also common among some invertebrate animals (worms, insects). Asexual reproduction in plants and invertebrates usually takes place by budding or splitting.

1.3 The first successful cloning in vertebrate animals 
was reported in 1952, in frogs. Nuclei from early frog embryos were transferred to unfertilised frog eggs from which the original nuclei had been removed. The resulting clones were not reared beyond the tadpole stage. In the $1960 \mathrm{~s}$, clones of adult frogs were produced by transfer not only of nuclei from early embryos but also of nuclei from differentiated larval intestinal cells. Later, clones of feeding tadpoles were obtained by nuclear transfer from differentiated adult cells, establishing that differentiation of cells involving selective gene expression does not require the loss or irreversible inactivation of genes. Nuclear transfer in frogs has not yet generated an adult animal from cells of an adult animal.

1.4 Nuclear transfer can be used for different objectives. Nuclear transfer in mice has been used to show that both a female and male set of genes are required for development to birth. If the two pronuclei, taken from fertilised eggs and transferred into an enucleated egg, are only maternal or only paternal, normal development does not occur. This is not cloning, since the single embryo formed is not identical to any other embryo and the objective is not to multiply individuals.

1.5 Nuclear transfer has also been used for cloning in various mammalian species (mice, rabbits, sheep, cattle), but until recently only nuclei taken from very early embryos were effective, and development was often abnormal, for reasons that are not fully understood.

1.6 In contrast, cloning by embryo splitting, from the 2-cell up to the blastocyst stage, has been extensively used in sheep and cattle to increase the yield of progeny from genetically high-grade parents. Because of the different pattern of early development, embryo splitting is much less successful in mice. From a scientific point of view, it would probably not be very effective in the human, although monozygotic (one-egg) twins and higher multiples occur naturally at a low incidence.

1.7 In 1996, a new method of cloning sheep embryos was reported, which involved first establishing cell cultures from single embryos. Nuclei from the cultured cells were transferred to enucleated unfertilised sheep eggs, particular attention being paid to the cell cycle stage of both donor and host cells, and the eggs were then artificially stimulated to develop. Genetically identical normal lambs were born.

1.8 Cell cultures were then established not only from embryonic and fetal stages, but also from mammary tissue taken from a 6-year-old sheep. Nuclear transfer was carried out as before, and in 1997 it was reported that several lambs had been born from the embryonic and fetal transfers and one lamb named
Dolly (out of 277 attempts) from the adult nucleat? transfer. It is not known whether the transferreof. nucleus was from a differentiated mammary gland? cell or from a stem cell.

1.9 From the point of view of basic research, this result is important. If repeatable it may allow greate insight into the aging process, how much is due tor cell aging, and whether or not it is reversible. Such work may also increase our understanding of celf commitment, the origin of the cancer process, and $b$ whether it can be reversed, but at the present timethe research is at a very early stage. Dolly may have w shortened lifespan or a greater susceptibility to cancer: if she is fertile, her progeny may show anp increased abnormality rate, owing to the accumulaw tion of somatic mutations and chromosomabs damage.

CONCERNING THE APPLICATIONS OF ANIMAL CLONING

1.10 Potential uses of cloning animals are reportedo to include:

- in the field of medicine and medical research, to늠 improve genetic and physiological knowledge, to make models for human diseases, to produce of lower cost proteins like milk proteins to be used therapeutic aims, to provide a source of organs tissues for xenotransplantation;

- in agriculture and agronomical research, tő improve the selection of animals or to reproduce animals having specific qualities (longevity, resis $\overrightarrow{\overrightarrow{0}}$ tance, ...) either innate, or acquired by transgenesis

1.11 From the point of view of animal breeding, the technology could be useful, in particular if it increases the medical and agricultural benefits expected from transgenesis (genetic modification of animals). By using genetic modification and selec-i tion in cultured cell lines, rather than in adulis animals, it could become possible to remove genes, such as those provoking allergic reactions, as well as adding genes, for the benefit of human health.

1.12 Furthermore, transgenesis is an uncertaino process: different transgenic animals express tho introduced gene in a different manner and to a diffN ferent extent, and do not "breed true". Cloning of adult animals of high performance, if it is possible would reduce the number of transgenic animalse needed and would allow human pharmaceuticals $\$$ for example, to be produced at a lower cost than would be possible otherwise.

1.13 If the use of cloning became more widespreado in the animal breeding industry, for example to bring the level of the general herd up to the level of the elite breeding populations, there is a danger that the 
level of genetic diversity could fall to an unacceptable degree. The introduction of artificial insemination in cattle raised similar problems.

\section{CONCERNING HUMAN IMPLICATIONS}

1.14 A clear distinction must be drawn between reproductive cloning aimed at the birth of identical individuals, which in humans has never been performed, and non-reproductive cloning, limited to the in vitro phase.

1.15 In considering human implications, we must again distinguish between cloning by embryo splitting and cloning by nuclear replacement (see 1.1). We must also distinguish between nuclear replacement as a means of cloning and nuclear replacement as a therapeutic measure, for example to avoid the very serious consequences of mitochondrial disease. The latter situation, which would require an enucleated donor egg containing normal mitochondria, as it need not involve the production of genetically identical individuals, will not be considered further here although we appreciate that it will raise ethical problems of its own.

1.16 Embryo splitting in the human is the event that gives rise to monozygotic (one-egg) twins and higher multiples. It has been discussed in the context of assisted reproduction, as a means of increasing the success rate of IVF, but there is no evidence that it has ever been used for this purpose, nor that it would be effective if it were so used, because of the pattern of early development of the human embryo.

1.17 Monozygotic twins show us that genetically identical individuals are far from identical: they may differ from one another not only physically but also psychologically, and in terms of personality. Individuals cloned by nuclear transfer from an adult cell would of course be even more different from their donor, since they would have different mitochondrial populations, they would be different in age and they would have had a different environment both before and after birth and a different upbringing. We are not just our genes.

1.18 There is no ethical objection to genetically identical human beings per se existing, since monozygotic twins are not discriminated against. However, the use of embryo splitting, or the use of human embryo cells as nuclear donors, deliberately to produce genetically identical human beings raises serious ethical issues, concerned with human responsibility and instrumentalization of human beings.

1.19 However, research involving human nuclear transfer could have important therapeutic implications, for example the development of appropriate stem cell cultures for repairing human organs. It could also provide insights into how to induce regeneration of damaged human tissues. If such research resulted in embryonic development, serious and controversial ethical issues concerning human embryo research would of course arise. Any attempt to develop methods of human reproductive cloning would require a large amount of human experimentation.

1.20 If adult cells were to be used as nuclear donors, we are still ignorant of the possible risks: whether the cloned individuals would have a shorter life-span, a greater susceptibility to cancer, whether they would be fertile, and if so whether they or their offspring would suffer from an abnormal rate of genetic abnormalities. Furthermore, the procedure would be immensely costly: each attempt would require several eggs and an available uterus, and many attempts would be unsuccessful. The issues of human responsibility and instrumentalization of human beings are even more ethically acute in this context.

\section{The group submits the following Opinion to the European Commission}

CONCERNING CLONING OF ANIMALS

2.1 Research on cloning in laboratory and farm animals is likely to add to our understanding of biological processes, in particular aging and cell commitment, and hence may contribute to human wellbeing. It is ethically only acceptable if carried out with strict regard to animal welfare, under the supervision of licensing bodies.

2.2 Cloning of farm animals may prove to be of medical and agricultural as well as economic benefit. It is acceptable only when the aims and methods are ethically justified and when it is carried out under ethical conditions, as outlined in the GAEIB's Opinion no 7 on the Genetic Modification of Animals.

\subsection{These ethical conditions include:}

- the duty to avoid or minimise animal suffering since unjustified or disproportionate suffering is unacceptable;

- the duty of reducing, replacing and when possible refining the experimentation adopted for the use of animals in research;

- the lack of better alternatives;

- human responsibility for animals, nature and the environment, including biodiversity.

2.4 Particular attention should be paid to the need to preserve genetic diversity in farm animal stocks. 
Strategies to incorporate cloning into breeding schemes while maintaining diversity should be developed by European institutions.

2.5 In so far as cloning contributes to health, special attention should be paid to the public's right to protection against risks as well as their right to adequate information. Furthermore, if the costs of production are reduced, consumers should also benefit.

\section{CONCERNING HUMAN IMPLICATIONS}

2.6 As far as reproductive cloning is concerned, many motives have been proposed, from the frankly selfish (the elderly millionaire vainly seeking immortality) to the apparently acceptable (the couple seeking a replacement for a dead child, or a fully compatible donor for a dying child, or the attempt to perpetuate some extraordinary artistic or intellectual talent). Considerations of instrumentalization and eugenics render any such acts ethically unacceptable. In addition, since these techniques entail increased potential risks, safety considerations constitute another ethical objection. In the light of these considerations, any attempt to produce a genetically identical human individual by nuclear substitution from a human adult or child cell ("reproductive cloning") should be prohibited.

2.7 The ethical objections against cloning also rule out any attempt to make genetically identical embryos for clinical use in assisted reproduction, either by embryo splitting or by nuclear transfer from an existing embryo, however understandable.

2.8 Multiple cloning is a fortiori unacceptable. In any case, its demands on egg donors and surrogate mothers would be outwith the realms of practicality at the present time.

2.9 Taking into account the serious ethical controversies surrounding human embryo research: for those countries in which non-therapeutic research on human embryos is allowed under strict licence, a research project involving nuclear substitution should have the objective either to throw light onm the cause of human disease or to contribute to the alleviation of suffering, and should not includes? replacement of the manipulated embryo in a uterus $\overrightarrow{\overrightarrow{\vec{F}}}$

2.10 The European Community should clearly음 express its condemnation of human reproductive cloning and should take this into account in the relevant texts and regulations in preparation as the Decision adopting the Vth Framework Programmes for Research and Development (1998-2002) and the proposed directive on legal protection of biotechnological inventions.

\section{GENERAL REMARKS}

2.11 Further efforts must be made to inform the public, to improve public awareness of potentiabs risks and benefits of such technologies, and to fosteri informed opinion. The European Commission ise invited to stimulate the debate involving the public, 욱 consumers, patients, environment and animal pro- tection associations, and a well-structured public debate should be set up at European level Universities and high schools should also be involved in the debate at European level.

2.12 These new technologies increase the powerofo people over nature and thus increase their respons:bilities and duties. Along the line of the promotion by the European Commission of research on thes ethical, legal and social aspects of life sciences, the commission should continue to foster ethica research on cloning related areas, at a European? level.

In accordance with its mandate, the Group of Advisers on the Ethical Implications of $\mathrm{Bio}_{-}^{-}$ technology submits this Opinion to the European Commission.

The members: Anne McLaren, Margareta Mikkelsen, Luis Archer; Octavi Quintana; Stefano Rodota; Egber Schroten; Dietmar Mieth; Gilbert Hottois; Chairman Noëlle Lenoir. 